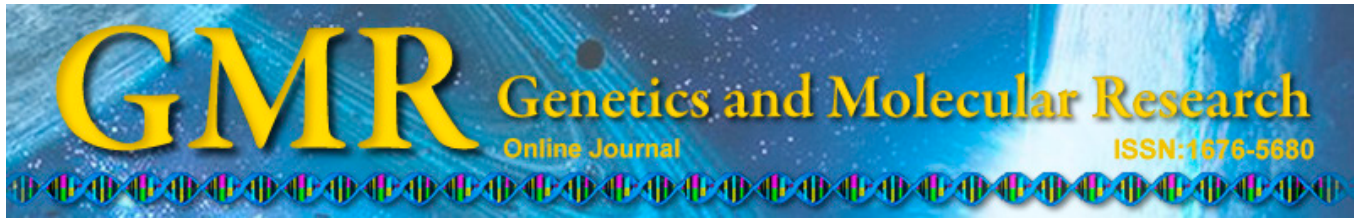

\title{
Association between $X R C C 1$ Arg280His polymorphism and risk of hepatocellular carcinoma: a systematic review and meta-analysis
}

\author{
W. Xü ${ }^{2 *}$, S. Liu ${ }^{3 *}$, L. Li ${ }^{2}$, Z.Y. Shen ${ }^{4}$ and Y.L. Wu ${ }^{1}$ \\ ${ }^{1}$ Shanghai Key Laboratory of Regulatory Biology, \\ Institute of Biomedical Sciences and School of Life Sciences, \\ East China Normal University, Shanghai, China \\ ${ }^{2}$ Department of Bone Tumor, Changzheng Hospital, \\ Second Military Medical University, Shanghai, China \\ ${ }^{3}$ Department of Hematology, Guangdong No. 2 People's Provincial Hospital No. 1, \\ Guangzhou, China \\ ${ }^{4}$ Department of Electronic Engineering, City University of Hong Kong, \\ Hong Kong, China \\ *These authors contributed equally to this study. \\ Corresponding author: Y.L. Wu \\ E-mail: wy19900@163.com
}

Genet. Mol. Res. 14 (2): $7122-7129$ (2015)

Received June 26, 2014

Accepted January 22, 2015

Published June 29, 2015

DOI http://dx.doi.org/10.4238/2015.June.29.5

\begin{abstract}
Hepatocellular carcinoma (HCC) is one of the most lifethreatening malignancies worldwide. Defects in DNA repair genes may increase the risk of HCC. X-ray cross-complementing group 1 gene $(X R C C 1)$ is a major DNA repair gene involved in base excision repair. Recently, several studies have indicated that an association exists between XRCC1 polymorphism and HCC, particularly the Arg280His polymorphism. However, the data is inconsistent and incomplete. In this study, we conducted a meta-analysis to investigate the association
\end{abstract}


between the $\mathrm{XRCC1} \mathrm{Arg} 280 \mathrm{His}$ polymorphism and HCC risk. A total of 10 case-control studies included 1848 HCC cases and 1969 controls were examined in this analysis. Our results suggest that variant genotypes of the XRCC1 Arg280His gene are associated with a significantly increased risk of HCC in homozygote comparison (HisHis vs ArgArg, odds ratio, $1.55,95 \%$ confidence interval, $1.10-2.18, \mathrm{P}=0.013$ ); no heterogeneity was observed $\left(\mathrm{I}^{2}=0 \%\right)$. Our analysis suggests that the XRCC1 Arg280His polymorphism is associated with a higher risk of HCC.

Key words: Hepatocellular carcinoma; Meta-analysis; Single nucleotide polymorphism; XRCC1-Arg280His

\section{INTRODUCTION}

Hepatocellular carcinoma (HCC) is a major cancer worldwide because of its highgrade malignancy and lack of effectiveness of medical treatment. It is associated with poor prognosis, and its incidence is increasing in many countries (Siegel, et al., 2013). Hepatitis B virus infection is the predominant risk factor in Southeast Asia and China. However, hepatitis $\mathrm{C}$ virus infection has emerged as a more significant risk factor in Japan, North America, and Europe (Gomaa et al., 2008; Venook et al., 2010).

DNA is under constant threat from endogenous and exogenous DNA damaging agents. Mammalian cells have evolved highly conserved DNA repair systems to process DNA damage and maintain genomic integrity (Abbotts et al., 2014). These processes play a critical role in tumorigenesis and treatment resistance (Deans and West, 2011; Wilting and Dannenberg, 2012). Impaired DNA repair is a major factor in carcinogenesis and can promote aggressive cancer biology. The X-ray repair cross complementing group 1 (XRCC1), a key protein in the base excision repair system, serves as a scaffold protein for repairing base lesions induced by reactive oxygen species (David et al., 2007). More than 300 single-nucleotide polymorphisms have been identified in the XRCC1 gene in the dbSNP database, but only 3 have been extensively studied, including Arg280His, Arg194Trp, and Arg399Gln (Li et al., 2012). These nonconservative amino acid changes may alter the function of the XRCC1 protein, diminish repair kinetics, and result in altered efficiency of the protein, eventually inducing cancer development.

In recent years, the association between the XRCC1 Arg280His polymorphism and the risk of HCC has been investigated by several groups. However, because of limited sample sizes, varying geographic regions, and differences among research subjects, results regarding the association between the XRCC1 Arg280His polymorphism and the risk of $\mathrm{HCC}$ have been inconsistent (Kiran et al., 2009; Yuan et al., 2012a,b; Li et al., 2012, 2013a). In this study, we performed a meta-analysis to clarify the association between the XRCC1 Arg280His polymorphism and the risk of HCC. Our results suggest that the variant genotypes of the XRCC1 Arg280His gene are associated with a significantly increased risk of HCC in the homozygote comparison model.

\section{MATERIAL AND METHODS}

\section{Data collection}

For first-round exclusion, articles were searched in the NCBI Global Cross-data- 
base, including PubMed, PMC, Gene, PubChem, etc. using "DNA polymorphism", "hepatocellular carcinoma", "HCC", and "XRCC1" as key words. All articles were published in English. We examined all articles whose primary topic was the association between DNA repair gene $X R C C 1$ polymorphism and HCC and identified a total of 162 articles. The following inclusion criteria were specified: case-control design with genotyping of $\mathrm{HCC}$ and studies that covered the XRCC1 Arg280His polymorphism. We excluded studies that did not examine the association between hepatocellular carcinoma or did not include data for this specific DNA polymorphism. In cases of overlapping data, we retained that with the most extensive results. A total of 10 studies were included in our meta-analysis. The data collection flow chart is shown in Figure 1.

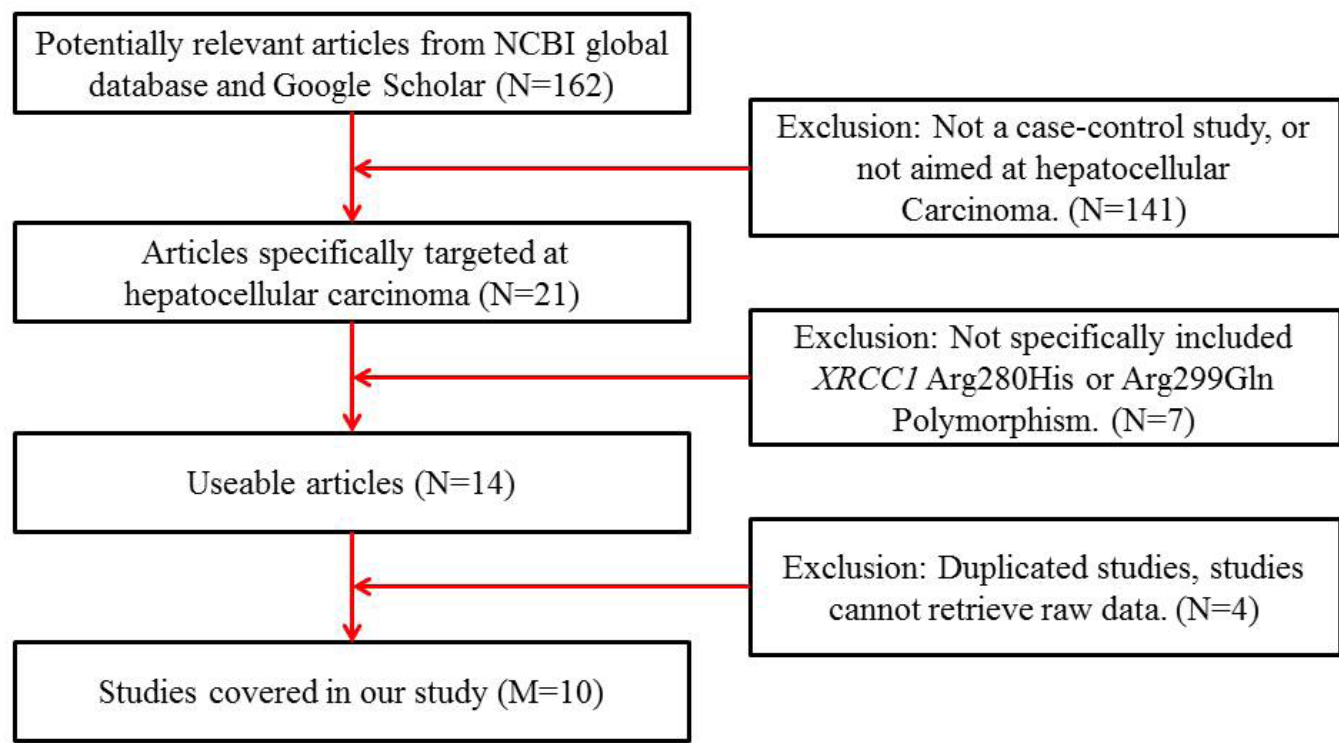

Figure 1. Flow chart of literature search and study selection. A total of 162 articles were searched for first-round exclusion. Ten studies were included in the final meta-analysis.

\section{Study selection}

Multiple electronic databases were searched for data collection. Ten case-control studies (1848 cases, 1969 controls) examining the Arg280His polymorphism were included in this meta-analysis. The association between the XRCC1 Arg280His polymorphism and HCC was evaluated using the odds ratio (OR) derived from the recessive model (HisHis vs ArgArg+ArgHis), dominant model (HisHis + ArgHis $v s$ ArgArg), and homozygote comparison (HisHis vs ArgArg). Statistical analysis was performed using STATA 12 version 12 (StataCorp, College Station, TX, USA). Heterogeneity was measured using the $\mathrm{I}^{2}$ index. To account for significant heterogeneity, different analysis models were used: Mantel-Haenszel (M-H) fixedeffect model and DerSimonian and Laird (D-L) random-effect model were applied for the Arg280His dataset. A forest plot and Begg's funnel plot were generated to evaluate OR and publication bias. To better analyze publication bias, Egger's test was also completed for each dataset. 


\section{Statistical analysis}

Three different models were examined, including the dominant model, recessive model, and homozygote comparison. In the dominant model, we used Arg/Arg as the reference genotype to investigate the distribution of the His/His $+\mathrm{Arg} / \mathrm{His}$ genotype. In the recessive model, we chose Arg/Arg+Arg/His as the reference genotype and investigated the distribution of the His/His genotype. In the homozygote comparison, we used Arg/Arg as the reference genotype and investigated the distribution of the His/His genotype. For each study, the numbers of the 3 genotypes in the case and control groups were used as pooled data. For large samples, Peto's method may be misleading. In contrast, inverse variance method is only effective when examining continuous data. Another key factor in choosing analysis model is the heterogeneity involved in the studies. The $\mathrm{M}-\mathrm{H}$ fixed-effect model can be applied to analyze datasets without significant heterogeneity, while the DerSimonian and Laird random-effects model should be applied for datasets showing obvious heterogeneity. In our analysis, the heterogeneity between studies was tested using the $\mathrm{I}^{2}$ index; in the equation, $\mathrm{Q}$ is statistical data and $\mathrm{df}$ is degrees of freedom. A higher $\mathrm{I}^{2}$ values indicates more significant heterogeneity. Values of $\mathrm{I}^{2}=25,50$, and $75 \%$ represent low, medium, and high heterogeneity, respectively. When $\mathrm{I}^{2} \leq 50 \%$, there is no significant heterogeneity between pooled data. In this meta-analysis, 10 studies were included in the final analysis for codon Arg280. First, we used the M-H fixedeffect model to test heterogeneity, and then used a different model based on these results. ORs were calculated with each model within $95 \%$ confidence intervals. The available polymorphism data were analyzed using the STATA 12 software. Forest plots were generated using 3 statistical methods (dominant, recessive, and homozygote) to summarize the results. To evaluate publication bias, Begg's funnel plots were generated based on the analysis results and database size. Higher asymmetry in the funnel plot indicates more publication biases. Egger's test was also performed for further investigation.

\section{RESULTS}

\section{Characteristics of studies included}

The search strategy retrieved 162 potentially relevant studies. According to the inclusion criteria, 10 studies with full-text were included in this meta-analysis (Kiran et al., 2009; Li et al., 2012; Xie et al., 2012; Duan et al., 2012; Yuan et al., 2012a,b; Han et al., 2012; Gulnaz et al., 2012) and 152 studies were excluded. The flow chart for study selection is summarized in Figure 1. These 10 case-control studies selected included a total of $1848 \mathrm{HCC}$ cases and 1969 healthy controls. All data in these studies were related to the association between the XRCC1 Arg280His gene polymorphism and HCC risk. The characteristics of studies included are shown in Table 1.

\section{Quantitative synthesis}

First, the M-H fixed-effects model was applied to the dataset using 3 models to analyze heterogeneity. The results are shown in Table 2. Based on the results, we selected different methods (M-H fixed effect model, DerSimonian and Laird random-effects model) for analysis.

The analysis results using 3 different methods for the Arg280His polymorphism are 
summarized in Table 3, including the results for the bias test. For the dominant model, the overall OR was $1.26(95 \%$ confidence interval, $0.95-1.68, \mathrm{P}=0.115)$. Because of significant heterogeneity $\left(\mathrm{I}^{2}=65.7 \%\right)$, we used the random-effect model for analysis. For the recessive model, the overall OR was 1.30 (95\% confidence interval, $0.94-1.80, \mathrm{P}=0.118)$, and no heterogeneity was observed $\left(\mathrm{I}^{2}=0 \%\right)$. For homozygote comparison, the overall OR was 1.55 ( $95 \%$ confidence interval, $1.10-2.18, \mathrm{P}=0.013)$, and no heterogeneity was observed $\left(\mathrm{I}^{2}=0 \%\right)$. The forest plots for all 3 models are shown in Figure 2. Based on homozygote comparison, the Arg280His showed an effect on HCC risk. These results showed that an association exists between the XRCC1 Arg280His polymorphism and HCC according to the homozygote comparison model. Although patients with His showed a relatively higher risk of HCC compared to patients with Arg in both the dominant and recessive model, with ORs of 1.26 and 1.30, respectively, the results were not statistically significant, and further investigation is required.

\begin{tabular}{|c|c|c|c|c|c|c|c|c|}
\hline \multirow[t]{2}{*}{ Study/Year } & \multicolumn{4}{|c|}{ Cases } & \multicolumn{4}{|c|}{ Controls } \\
\hline & Arg/Arg & Arg/His & His/His & Total & Arg/Arg & $\mathrm{Arg} / \mathrm{His}$ & His/His & Total \\
\hline Su 2008 & 79 & 20 & 1 & 100 & 87 & 21 & 3 & 111 \\
\hline Kiran 2009 & 17 & 30 & 14 & 50 & 91 & 29 & 35 & 155 \\
\hline Wu 2009 & 77 & 22 & 1 & 100 & 47 & 13 & 0 & 50 \\
\hline Zeng 2010 & 451 & 86 & 8 & 545 & 423 & 86 & 6 & 515 \\
\hline Tang 2011 & 138 & 11 & 1 & 150 & 123 & 26 & 1 & 150 \\
\hline Yuan 2012a & 272 & 73 & 5 & 350 & 329 & 64 & 7 & 400 \\
\hline Во 2012 & 64 & 12 & 8 & 84 & 78 & 9 & 3 & 90 \\
\hline Han 2012 & 72 & 47 & 31 & 150 & 84 & 47 & 28 & 159 \\
\hline Yuan 2012b & 193 & 59 & 6 & 258 & 206 & 44 & 5 & 255 \\
\hline Gulnaz 2013 & 24 & 17 & 9 & 50 & 329 & 64 & 7 & 400 \\
\hline
\end{tabular}

\section{Table 2. Heterogeneity test.}

\begin{tabular}{lccc}
\hline Datasets & Heterogeneity $\left(\mathrm{I}^{2}\right)$ & P value & Suggested Model \\
\hline Arg280His Dominant & $65.7 \%$ & 0.002 & Random-effects model \\
Arg280His Recessive & $0 \%$ & 0.543 & Fixed-effects model \\
Arg280His Homozygote & $0 \%$ & 0.517 & Fixed-effects model \\
\hline
\end{tabular}

\section{Publication bias}

The funnel plot, Begg's test, and Egger's test were used to assess the publication bias of studies included. The funnel plot showed some scattering, which may indicate publication bias in this dataset because of the smaller sample size (Figure 3). Begg's and Egger's test results revealed no major publication bias in the present meta-analysis (Table 3).

Table 3. Analysis results for Arg280His polymorphism.

\begin{tabular}{lllccccc}
\hline Method & Genotype & Reference & Overall OR & 95\%CI & P value & Begg's P value & Egger's P value \\
\hline Dominant & His/His+Arg/His & Arg/Arg & 1.26 & $0.95-1.68$ & 0.115 & 0.721 & 0.683 \\
Recessive & His/His & Arg/His+Arg/Arg & 1.30 & $0.94-1.80$ & 0.118 & 0.592 & 0.705 \\
Homozygote & His/His & Arg/Arg & 1.55 & $1.10-2.18$ & 0.013 & 0.592 & 0.953 \\
\hline
\end{tabular}




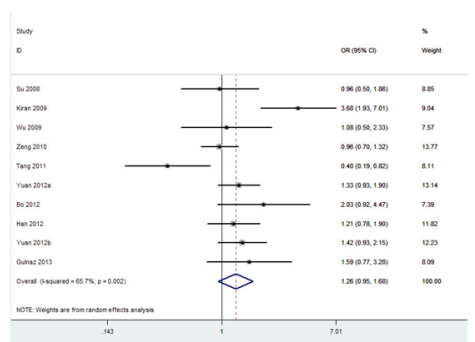

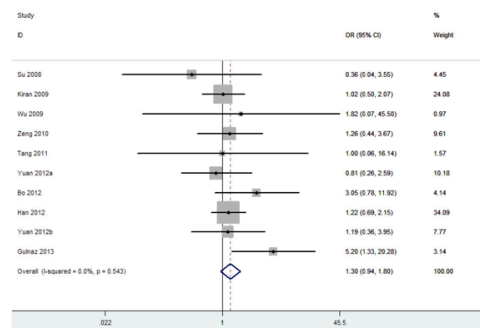

C

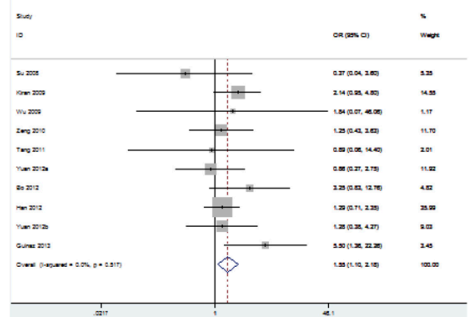

Figure 2. Forest plot of $X R C C 1 \mathrm{Arg} 280 \mathrm{His}$ polymorphism and the risk of $\mathrm{HCC}$ in the (A) dominant genetic model (HisHis+ArgHis $v s$ ArgArg), (B) recessive model (HisHis vs ArgArg+ArgHis), and (C) homozygote comparison (HisHis vs ArgArg). The center of each square represents the OR, the horizontal line indicates the 95\%CI. OR, odds ratio; CI, confidence interval; weights are from random-effects analysis for the dominant model; weights are from the fixed-effects analysis for the recessive model and homozygote comparison.

A
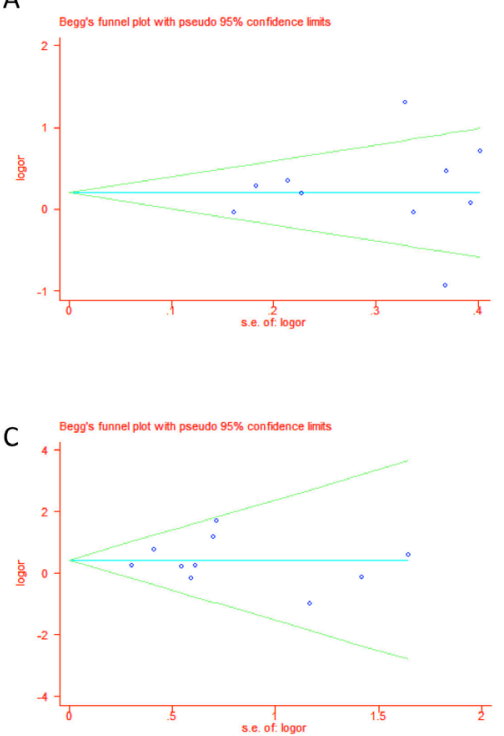

B Beg's funnel plot with pseudo $95 \%$ confidence Imits

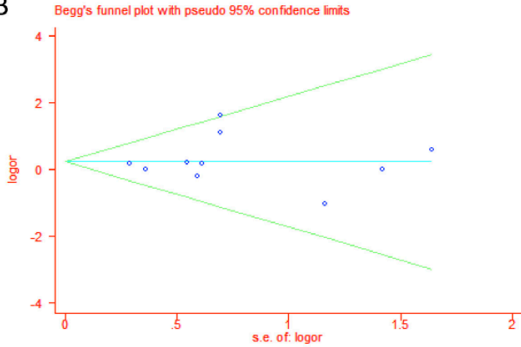

Figure 3. Funnel plot of XRCCl Arg280His polymorphism and HCC risk for dominant model (A), recessive model (B), and homozygote comparison (C). Each point represents a separate study for the indicated association. To determine publication bias in studies regarding the relationship between the XRCC1 Arg280His polymorphism and HCC susceptibility, a funnel plot with pseudo- $95 \%$ confidence limits was used. 


\section{DISCUSSION}

In this meta-analysis, we reviewed published studies that examined the association between the XRCC1 Arg280His polymorphism and susceptibility to HCC, which included a total of 1848 patients and 1969 controls in 10 studies. The overall data showed that the XRCC1 Arg280His polymorphism was associated with an increased risk of HCC.

$\mathrm{XRCC} 1$ is an indispensable protein involved in the DNA repair process and plays an important role in base-excision repair (Parsons et al., 2010), homologous recombination (Fan et al., 2007), and genome stability as well as the pathogenesis and progression of human cancers (Figueiredo et al., 2004; Canalle et al., 2006). Polymorphisms that alter XRCC1 expression and function may contribute to cancer risk (Goode et al., 2002; Hu et al., 2005). The XRCC1 Arg280His polymorphism is one of the most widely studied singlenucleotide polymorphisms and contributes to altered DNA repair capacity. Over the past 5 years, the XRCC1 Arg280His polymorphism has been shown to be associated with the susceptibility to HCC. However, because of small sample sizes and difference in research subjects, previous studies provided limited information and showed controversial results. Therefore, a systematic meta-analysis of the available studies may provide more definitive answers.

Meta-analysis is a useful tool for investigating the association between genetic variants and cancer risk as it can combine the results of similar studies on the same topic in a quantitative manner (Kirkham et al., 2012). To date, a total of 3 published meta-analyses have been performed to investigate the association between the XRCC1 Arg280His polymorphism and the risk of $\mathrm{HCC}$ (Li et al., 2012, 2013a,b). However, the results of these meta-analyses are either controversial or inconclusive. Li et al., 2012, 2013a,b) found no relationship between the XRCC1 Arg280His polymorphism and the risk of HCC. In contrast, Xie et al. (2012) reported that the XRCC1 Arg280 His polymorphism found a significant association between the His/His genotype and an increased risk of HCC (His/His vs Arg/Arg model, OR: 1.96, 95\%CI: $1.03-3.75, \mathrm{P}=0.04)$. Thus, we collected a larger sample of studies for a meta-analysis to evaluate the association between the XRCC1 Arg280His polymorphism and the risk of HCC. Our results indicated that the $X R C C 1 \mathrm{Arg} 280 \mathrm{His}$ polymorphism is associated with an increased risk of HCC under the homozygote comparison model.

There were some limitations to our meta-analysis. First, selection bias may have played a role because all studies selected in this meta-analysis were published in English, and other studies that may have influenced the evaluation were excluded. Second, although all cases and controls in each study were well-defined with similar inclusion criteria, there may be factors that were not taken into account that could have influenced our results. Finally, the sample size of some subgroups in the stratified analyses was limited, which may have reduced the power of these analyses. Thus, further studies with large sample sizes and well-designed multicenter analyses are required to clarify the association between $X R C C 1$ genetic polymorphisms and the risk of HCC.

\section{ACKNOWLEDGMENTS}

Research supported by the National Natural Science Foundation of China (grant \#81202327). 


\section{REFERENCES}

Abbotts R, Thompson N and Madhusudan S (2014). DNA repair in cancer: emerging targets for personalized therapy. Cancer Manag. Res. 6: 77-92.

Canalle R, da Silva Andrade V, Scrideli CA, de Paula Queiroz RG, et al. (2006). Polymorphisms in the thymidylate synthase promoter and the DNA repair genes XRCC1 and XPD in a Brazilian population. Environ. Mol. Mutagen. 47: 725-732.

David SS, O'Shea VL and Kundu S (2007). Base-excision repair of oxidative DNA damage. Nature 447: 941-950.

Deans AJ and West SC (2011). DNA interstrand crosslink repair and cancer. Nat. Rev. Cancer 11: 467-480.

Duan WH, Zhu ZY, Liu JG, Dong MS, et al. (2012). XRCC1 Arg399Gln gene polymorphism and hepatocellular carcinoma risk in the Chinese Han population: a meta-analysis. Asian Pac. J. Cancer Prev. 13: 3601-3604.

Fan J, Wilson PF, Wong HK, Urbin SS, et al. (2007). XRCC1 down-regulation in human cells leads to DNA-damaging agent hypersensitivity, elevated sister chromatid exchange, and reduced survival of BRCA2 mutant cells. Environ. Mol. Mutagen. 48: 491-500.

Figueiredo JC, Knight JA, Briollais L, Andrulis IL, et al. (2004). Polymorphisms XRCC1-R399Q and XRCC3-T241M and the risk of breast cancer at the Ontario site of the Breast Cancer Family Registry. Cancer Epidemiol. Biomarkers Prev. 13: 583-591.

Gomaa AI, Khan SA, Toledano MB, Waked I, et al. (2008). Hepatocellular carcinoma: epidemiology, risk factors and pathogenesis. World J. Gastroenterol. 14: 4300-4308.

Goode EL, Ulrich CM and Potter JD (2002). Polymorphisms in DNA repair genes and associations with cancer risk. Cancer Epidemiol. Biomarkers Prev. 11: 1513-1530.

Gulnaz A, Sayyed AH, Amin F, Khan Au, et al. (2012). Association of XRCC1, XRCC3, and XPD genetic polymorphism with an increased risk of hepatocellular carcinoma because of the hepatitis B and C virus. Eur. J. Gastroenterol. Hepatol. 25: 166-179.

Han X, Xing Q, Li Y, Sun J, et al. (2012). Study on the DNA repair gene XRCC1 and XRCC3 polymorphism in prediction and prognosis of hepatocellular carcinoma risk. Hepatogastroenterology 59: 2285-2289.

Hu Z, Ma H, Chen F, Wei Q, et al. (2005). XRCC1 polymorphisms and cancer risk: a meta-analysis of 38 case-control studies. Cancer Epidemiol. Biomarkers Prev. 14: 1810-1818.

Kiran M, Saxena R, Chawla YK and Kaur J (2009). Polymorphism of DNA repair gene XRCC1 and hepatitis-related hepatocellular carcinoma risk in Indian population. Mol. Cell. Biochem. 327: 7-13.

Kirkham JJ, Riley RD and Williamson PR (2012). A multivariate meta-analysis approach for reducing the impact of outcome reporting bias in systematic reviews. Stat. Med. 31: 2179-2195.

Li Y, Liu F, Tan SQ, Wang Y, et al. (2012). X-ray repair cross-complementing group 1 (XRCC1) genetic polymorphisms and cervical cancer risk: a huge systematic review and meta-analysis. PLoS One 7: e44441.

Li J, Li Z, Feng L, Guo W, et al. (2013a). Polymorphisms of DNA repair gene XRCC1 and hepatocellular carcinoma risk among East Asians: a meta-analysis. Tumor Biol. 34: 261-269.

Li LP, Wu W, Li XH and Song SS (2013b). The XRCC1 Arg280His gene polymorphism and hepatocellular carcinoma risk: a meta-analysis. Asian Pac. J. Cancer Prev. 14: 2033-2036.

Parsons JL, Dianova, II, Finch D, Tait PS, et al. (2010). XRCC1 phosphorylation by CK2 is required for its stability and efficient DNA repair. DNA Repair (Amst.) 9: 835-841.

Siegel R, Naishadham D and Jemal A (2013). Cancer statistics. CA Cancer J. Clin. 63: 11-30.

Venook AP, Papandreou C, Furuse J and de Guevara LL (2010). The incidence and epidemiology of hepatocellular carcinoma: a global and regional perspective. Oncologist 15 (Suppl 4): 5-13.

Wilting RH and Dannenberg JH (2012). Epigenetic mechanisms in tumorigenesis, tumor cell heterogeneity and drug resistance. Drug Resist. Updat. 15: 21-38.

Xie T, Wang ZG, Zhang JL and Liu H (2012). X-ray repair cross-complementing group 1 polymorphisms and hepatocellular carcinoma: a meta-analysis. World J. Gastroenterol. 18: 4207-4214.

Yuan T, Deng S, Liu H, Liu M, et al. (2012a). Relationship between XRCC1 and XPD polymorphisms and the risk of the development of hepatocellular carcinoma: A case-control study. Exp. Ther. Med. 4: 285-290.

Yuan T, Wei J, Luo J, Liu M, et al. (2012b). Polymorphisms of base-excision repair genes hOGG1 326cys and XRCC1 280His increase hepatocellular carcinoma risk. Dig. Dis. Sci. 57: 2451-2457. 